\title{
Modified reverse tapering method to prevent frequency shift of the radiation in the planar undulator
}

\author{
Chi Hyun Shim, ${ }^{1}$ In Soo Ko, ${ }^{1}$ and Yong Woon Parc ${ }^{2, *}$ \\ ${ }^{1}$ Department of Physics, Pohang University of Science and Technology, Pohang 37673, Korea \\ ${ }^{2}$ Pohang Accelerator Laboratory, Pohang University of Science and Technology, Pohang 37673, Korea
}

(Received 24 July 2016; published 13 March 2017)

\begin{abstract}
This paper presents a modified reverse tapering method to generate a polarized soft x ray in x-ray freeelectron lasers (XFELs) with a higher photon power and a shorter undulator length than the simple linear reverse tapering method. In the proposed method, a few untapered planar undulators are added before the simple linear reverse tapering section of the undulator line. This simple modification prevents the frequency shift of the radiation that occurs when the simple linear reverse tapering method is applied to planar undulators. In the proposed method, the total length of planar undulators decreased in spite of the additional untapered undulators. When the modified reverse tapering method is used with four untapered planar undulators, the total length of the planar undulators is $64.6 \mathrm{~m}$. On the other hand, the required length of the planar undulators is $94.6 \mathrm{~m}$ when the simple linear reverse tapering method is used. The proposed method gives us a way to generate a soft $\mathrm{x}$-ray pulse $(1.24 \mathrm{keV})$ with a high degree of polarization $(>0.99)$ and radiation power $(>30 \mathrm{GW})$ at the new undulator line with a $10-\mathrm{GeV}$ electron beam in the Pohang Accelerator Laboratory X-ray Free-Electron Laser. This method can be applied in the existing XFELs in the world without any change in the undulator lines.
\end{abstract}

DOI: 10.1103/PhysRevAccelBeams.20.030703

\section{INTRODUCTION}

$\mathrm{X}$-ray free-electron lasers (XFELs) based on self-amplified spontaneous emission (SASE) have been successfully operated [1-3] or will be operated within a few years [4-6]. At the Pohang Accelerator Laboratory X-ray FreeElectron Laser (PAL-XFEL), a SASE radiation from the undulator line was observed in June 2016. The saturation of the radiation power is expected to be achieved within 2016. Undulator lines in the PAL-XFEL undulator hall have a planar configuration in which linear polarization is the only option for the user service. However, polarization control in the soft $\mathrm{x}$-ray regime is essential for several kinds of experiments, so some alternatives [7-12] that use the existing planar undulator line have been proposed. The various methods for polarization control of a soft $\mathrm{x}$-ray FEL have been developed since practical $\mathrm{x}$-ray polarization optics is not available for the soft $\mathrm{X}$-ray region [13].

One of the alternatives is reverse tapering [12]. By using this method, the linearly polarized radiation power generated in a planar undulator line is suppressed by orders of magnitude, whereas the microbunching process of the electron beam is sufficiently progressed. The circularly polarized radiation power can be rapidly increased by placing only a small number of helical undulators after

\footnotetext{
*young1@postech.ac.kr
}

Published by the American Physical Society under the terms of the Creative Commons Attribution 3.0 License. Further distribution of this work must maintain attribution to the author(s) and the published article's title, journal citation, and DOI. the planar undulator line. These benefits can be obtained because the central frequency of the amplified radiation at the planar undulator line stays at the resonance frequency of the first undulator and does not shift along with the change of the resonance frequencies of the rest of the undulators [12].

However, practical results [14-16] differed from the prediction [12] and are more consistent with the explanation shown in Ref. [17] that the slowly varying reverse taper is the cause of the frequency shift from the resonance frequency of the first undulator to the mean value of the resonance frequencies between the first and the last undulators. This shift would be the origin of the inefficient growth of the bunching factor of the electron beam in the planar undulator line and also of the slow increase of the circularly polarized radiation power in the helical undulators.

In this paper, we propose a simple method to prevent the frequency shift by adding a number of untapered undulators in front of the simple linear reverse tapering method. Section II presents the configuration of the modified reverse tapering method and the simulation parameters. The simulation results are presented in Sec III. In Sec. IV, the effect of the frequency shift is analyzed, and it is demonstrated that our method can hold the radiation frequency close to the resonance frequency of the first undulator. Section V contains the conclusion and discussion.

\section{METHOD DESCRIPTION AND SIMULATION PARAMETERS}

The simple linear reverse tapering method presented in Ref. [12] for polarization control consists of two stages 


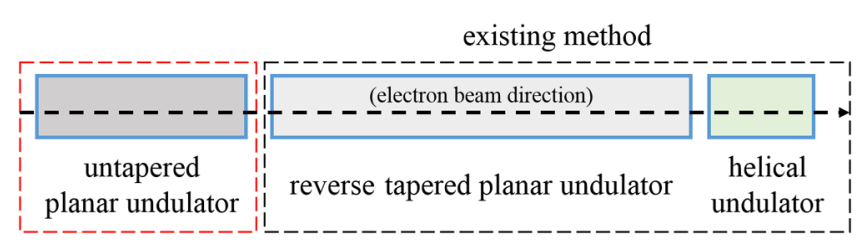

FIG. 1. Conceptual drawing of the modified reverse tapering method (combination of the red box and the black one) and the simple linear reverse tapering method (black box).

(Fig. 1, black box): the linearly reverse tapered planar undulator and the helical undulator. In the first stage, the linearly polarized radiation power increase is suppressed, but the bunching factor of the electron beam is still growing, as in the nontapering case. Then the circularly polarized radiation power increases quickly when the microbunched electron beam propagates the helical undulator. This method is quite simple, so it is easily adapted to existing or scheduled undulator lines. In this paper, this simple linear reverse tapering method is called "the existing method."

However, in the existing method, the central frequency of the amplified radiation at the planar undulator line shifts [14-16]. To prevent this shift, we added one more stage with the untapered planar undulator configuration (Fig. 1, red box) before the reverse tapered planar undulator. Our proposal (which is named "the modified reverse tapering method") can be described as the combination of the untapered planar undulators (red box) and the existing method (black box).

The parameters (Table I) for the PAL-XFEL [4] were used in the simulation. The undulator parameters in the table were also used in the proposal for the new undulator line with $10-\mathrm{GeV}$ electron beam energy in the PAL-XFEL [18].

TABLE I. Main parameters used in the simulations.

\begin{tabular}{lcc}
\hline \hline Parameter & Value & Unit \\
\hline Electron beam & & \\
Energy & 10 & $\mathrm{GeV}$ \\
Charge & 200 & $\mathrm{pC}$ \\
Peak current & 3 & $\mathrm{kA}$ \\
rms normalized slice emittance & 0.4 & $\mu \mathrm{m}$ \\
rms slice energy spread & 1.5 & $\mathrm{MeV}$ \\
Planar undulator & & \\
Period & 60 & $\mathrm{~mm}$ \\
$a_{w}$ & 3.43 & \\
Length & 4.98 & $\mathrm{~m}$ \\
Helical undulator & & \\
Period & 60 & $\mathrm{~mm}$ \\
$a_{w, \text { hel }}$ (existing method, $\left.N_{\mathrm{UT}}=0\right)$ & 3.442 & \\
(modified method, $\left.N_{\mathrm{UT}}=4\right)$ & 3.428 & \\
Length & 3.48 & $\mathrm{~m}$ \\
Radiation & & \\
Wavelength (photon energy) & $1(1240)$ & $\mathrm{nm}(\mathrm{eV})$ \\
Pierce parameter & $1.573 \times 10^{-3}$ & \\
\hline \hline
\end{tabular}

This new undulator line will generate radiation with photon energies from 1 to $2.5 \mathrm{keV}$, which is a vacant photon energy region in the current design. In the new undulator line, the length of the beam transport magnet lattice unit is $11.88 \mathrm{~m}$ (Fig. 2). The period of undulator $\lambda_{u}$ is $60 \mathrm{~mm}$. The length of the planar undulator is $83 \lambda_{u}=4.98 \mathrm{~m}$ [Fig. 2(a)]; the length of the helical undulator is $58 \lambda_{u}=3.48 \mathrm{~m}$ [Fig. 2(b)]. The drift space between two helical undulators is minimized to prevent the smearing out of the microbunching structure for the electron beam [Fig. 2(b)] [19].

\section{SIMULATION RESULTS}

Simulations considered six cases to compare the effects of the length of the untapered planar undulator: the existing method and five cases of the modified reverse tapering method (i.e., the number of untapered undulators $N_{\mathrm{UT}}=0$, $1,2,3,4$, or 5). The existing method will be expressed by $N_{\mathrm{UT}}=0$. Simulations were performed using GENESIS 1.3 [20], which is a time-dependent 3D FEL simulation code.

The taper strength $\beta$ in Fig. 3 is defined as [12]

$$
\beta=-\frac{\lambda_{u}}{4 \pi \rho^{2}} \frac{a_{w}(0)}{1+a_{w}(0)^{2}} \frac{d a_{w}}{d z},
$$

where $\lambda_{u}$ is the period of the undulator, $\rho$ is a FEL parameter (Pierce parameter), $a_{w}(0)$ is the initial rms undulator parameter, and $d a_{w} / d z$ is the derivative of the rms undulator parameter along the undulator line. Note that we use the rms undulator parameter $a_{w}=e \lambda_{u} B_{r m s} / 2 \pi m c$, which is given by the rms magnetic field strength $B_{r m s}$ with $B_{r m s}=B_{0} / \sqrt{2}$ for a planar undulator and $B_{r m s}=B_{0}$ for a helical undulator, where $B_{0}$ is the on-axis peak magnetic field strength [21]. In the reverse tapering configuration, the rms undulator parameter $a_{w}$ is increased along the undulator line, so the taper strength is negative.

To determine the appropriate taper strength, FEL simulations with planar undulators [Fig. 2(a)] were conducted by scanning $\beta$ (Fig. 3). The saturation point is defined as the point at which the bunching factor is highest. For the $\beta=0$ point, all cases in Fig. 3 result in the same configuration, i.e., normal SASE without any taper, and the linearly polarized radiation powers $\left(P_{\text {lin }}\right)$ for all cases are $30 \mathrm{GW}$ [Fig. 3(a)]. When a strong negative taper strength is applied to the planar undulator line, $P_{\text {lin }}$ at saturation was suppressed by orders of magnitude in all cases.

We did calculate a degree of circular polarization $\left|S_{3} / S_{0}\right|$ from the Stokes parameter [22] by using the linearly polarized radiation power $\left(P_{\text {lin }}\right)$ from the planar undulator line and the total radiation power $\left(P_{\text {tot }}\right)$ at the end of the helical undulator line (for the detailed derivation, see Ref. [23]):

$$
\left|\frac{S_{3}}{S_{0}}\right|=1-\frac{P_{\text {lin }}}{P_{\text {tot }}} .
$$




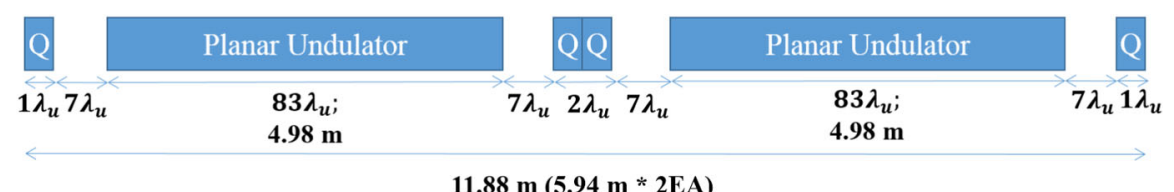

(a)

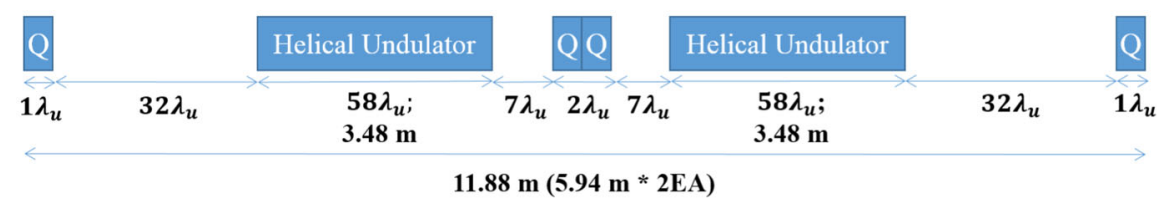

(b)

FIG. 2. The beam transport magnet lattice unit in the PAL-XFEL undulator line: (a) planar undulator line and (b) helical undulator line. $Q$ means a half part of a quadrupole magnet. $\lambda_{u}$ is the undulator period.

The degree of circular polarization can be mainly lowered due to linearly polarized radiation from the planar undulator line. The other possible contributions (field imperfections of the helical undulator, coherent synchrotron radiation, etc.) can also be causes of the decreasing. In this paper, however, these possible contributions were ignored, since those can be only a few per mil level, which is one order of magnitude lower than $P_{\text {lin }} / P_{\text {tot }}$ [12]. Our target $\left|S_{3} / S_{0}\right|$ is 0.99 ; therefore, the relation between the linear and circular power are given by

$$
P_{\text {lin }} \leq 0.01 P_{\text {tot }} .
$$

Equation (3) means that the linearly polarized radiation power from the planar undulator line must be suppressed to less than $1 \%$ of $P_{\text {tot }}$ at the end of the helical undulator line. $P_{\text {tot }}$ from the helical undulator line with the modified reverse tapering method was roughly $30 \mathrm{GW}$ (Fig. 4), so the $P_{\text {lin }}$ should be less than $0.3 \mathrm{GW}$ [dashed line, Fig. 3(a)]. The appropriate taper strengths of the five cases $\left(\beta\right.$ at which $\left.P_{\text {lin }}=0.3 \mathrm{GW}\right)$ were determined by using the plots of $P_{\text {lin }}$ vs $\beta$. When the existing method $\left(N_{\mathrm{UT}}=0\right)$ was used, the appropriate $\beta$ was $-0.71[(0)$, Fig. 3(a)], and the linearly polarized radiation power $\left(P_{\text {lin }}\right)$ was $0.29 \mathrm{GW}$. When the modified reverse tapering method was used, the appropriate $\beta$ were -0.75 for $N_{\mathrm{UT}}=1\left(P_{\text {lin }}=0.30 \mathrm{GW}\right),-0.84$ for $N_{\mathrm{UT}}=2\left(P_{\text {lin }}=\right.$ $0.30 \mathrm{GW}),-1.08$ for $N_{\mathrm{UT}}=3\left(P_{\mathrm{lin}}=0.29 \mathrm{GW}\right)$, and -1.40 for $N_{\mathrm{UT}}=4 \quad\left(P_{\text {lin }}=0.30 \mathrm{GW}\right) \quad[(1),(2), \quad(3)$, and (4) in Fig. 3(a)]. The appropriate $\beta$ for the case of $N_{\text {UT }}=5$ cannot be determined, because the linearly polarized radiation power at the end of untapered section is already $0.39 \mathrm{GW}$, which exceeds over our target linearly polarized radiation power. The undulator parameter $\left(a_{w}\right)$ of each planar undulator increased linearly from its initial value of 3.43 along the planar undulator line from the $\left(N_{\mathrm{UT}}+1\right)$ th undulator. The slope of the line increased as $N_{\mathrm{UT}}$ increased [Fig. 3(b)].

At all $N_{\mathrm{UT}}$, the bunching factor at saturation decreased as the amplitude of the taper strength increased and in all cases was over 0.38 [Fig. 3(c)]. Generally, the saturation length $L_{\text {sat }}$ increased as the amplitude of the taper strength increased [Fig. 3(d)]. However, we need to compare the five simulation points determined in Fig. 3(a), because only the five points satisfy Eq. (3). As we can see in Fig. 3(d), $L_{\text {sat }}$ decreased as $N_{\mathrm{UT}}$ increased [see the black arrows, i.e., from $94.6 \mathrm{~m}$ at $N_{\mathrm{UT}}=0$ (the existing method), $89.2 \mathrm{~m}$ at $N_{\mathrm{UT}}=1,81.7 \mathrm{~m}$ at $N_{\mathrm{UT}}=2,72.8 \mathrm{~m}$ at $N_{\mathrm{UT}}=3$, and $64.6 \mathrm{~m}$ at $\left.N_{\mathrm{UT}}=4\right]$. These results show that the modified reverse tapering method costs less than the existing method to realize the same degree of circular polarization.

The total radiation power $\left(P_{\text {tot }}\right)$ and the degree of circular polarization $\left(\left|S_{3} / S_{0}\right|\right)$ at the end of the helical undulator line for the selected cases were simulated by scanning the rms undulator parameter of the helical undulator $a_{w \text {,hel }}$ (Fig. 4). In the existing method, the $P_{\text {tot }}$ reached only $16.7 \mathrm{GW}$ [black line, Fig. 4(a)] and $\left|S_{3} / S_{0}\right|=0.983$ [black line, Fig. 4(b)] at $a_{w, \text { hel }}=3.442$. In the modified reverse tapering method, the total radiation powers were all higher than the existing method. When $N_{\mathrm{UT}}=1$ (magenta line), $P_{\text {tot }}$ reached $20.8 \mathrm{GW}$ and $\left|S_{3} / S_{0}\right|=0.986$ at $a_{w \text {,hel }}=$ 3.434. With $N_{\mathrm{UT}}=2$ (red line), $P_{\text {tot }}$ reached $24.7 \mathrm{GW}$ and $\left|S_{3} / S_{0}\right|=0.988$. For $N_{\mathrm{UT}}=3$ (green line), $P_{\text {tot }}$ reached $30.5 \mathrm{GW}$ and $\left|S_{3} / S_{0}\right|=0.990$. When $N_{\mathrm{UT}}=4$ (blue line), $P_{\text {tot }}$ reached $31.2 \mathrm{GW}$ and $\left|S_{3} / S_{0}\right|=0.990$ at the end of the helical undulator line. For the cases $N_{\mathrm{UT}} \geq 2$, the maxima occurred at $a_{w}$,hel $=3.428$, which is almost the same value with the $a_{w}$ of the first undulator in the planar undulator line. Note that the helical undulator parameter $a_{w, \text { hel }}$ is slightly smaller than the undulator parameter for the first planar undulator, because there was a radiation from the electron beam in the planar undulator which made the energy loss in the electrons in the electron beam.

In the existing method (Fig. 1, black box), the rms undulator parameter of the helical undulator $a_{w \text {,hel }}$ is between the rms undulator parameters of the first planar undulator and the final one [14,15]. The rms undulator parameter for the helical undulator $a_{w, \text { hel }}$ with the existing method to get the maximum $P_{\text {tot }}$ is 3.442 [Fig. 4(a)], which 


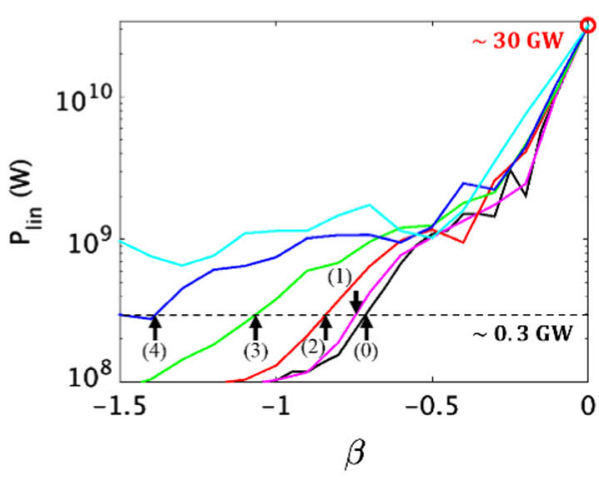

(a)

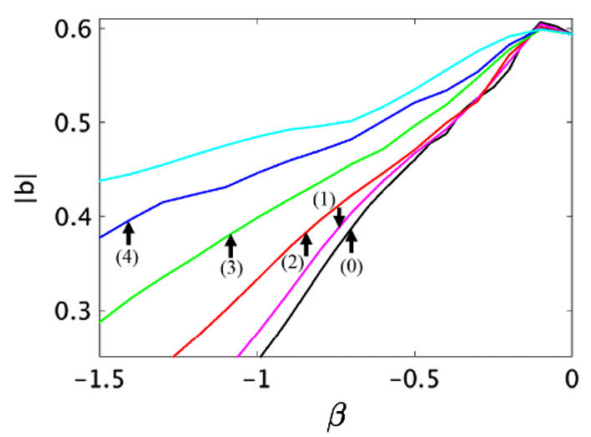

(c)

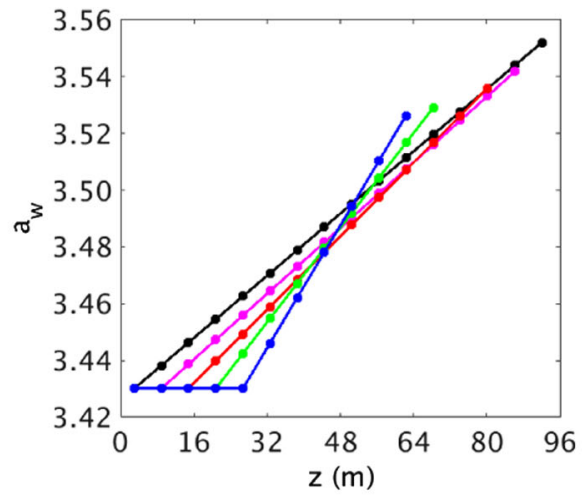

(b)

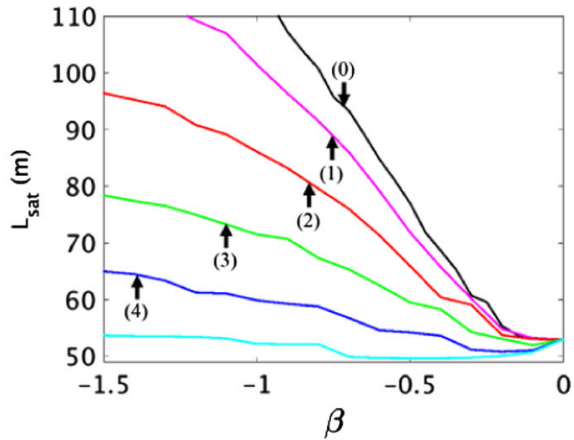

(d)

FIG. 3. Simulation results of the existing method (black lines) and modified reverse tapering method with 1 (magenta lines), 2 (red lines), 3 (green lines), 4 (blue lines), and 5 (cyan lines) untapered planar undulators: (a) Linearly polarized radiation power at the saturation point vs the taper strength. The dotted line denotes the target radiation power to be controlled. The black arrows with a number indicate the cross points between the solid lines and the dotted line to determine the taper strength for each case. (b) The rms undulator parameters along the planar undulator line for the five cases with the taper strength denoted by the arrows in (a). Note that the taper strengths are applied after the untapered undulators for each case. (c) Bunching factor at saturation point vs the taper strength. (d) Saturation length vs the taper strength. The black arrows in (c) and (d) indicate the same simulation points determined in (a).

is higher than the rms undulator parameter of the first planar undulator. This means that the central frequency of the radiation did not stay at the resonance frequency of the first undulator but shifted along with the change of the resonance frequencies for the rest of the undulators.
However, when the modified reverse tapering method was used, $a_{w, \text { hel }}$ to get the maximum $P_{\text {tot }}$ got into close touch with the $a_{w}$ of the first planar undulator. These results signify that the central frequency of the radiation remains at the resonance frequency of the first undulator in the planar

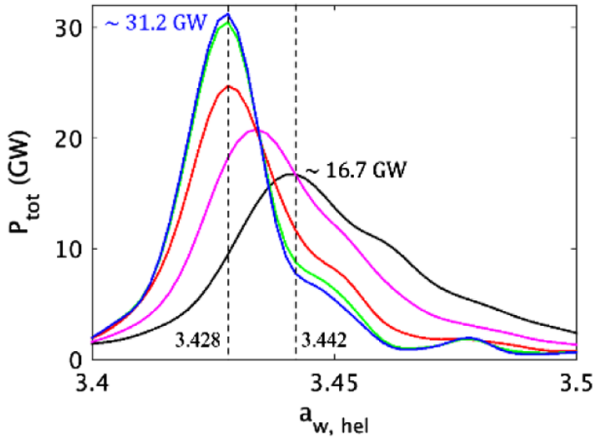

(a)

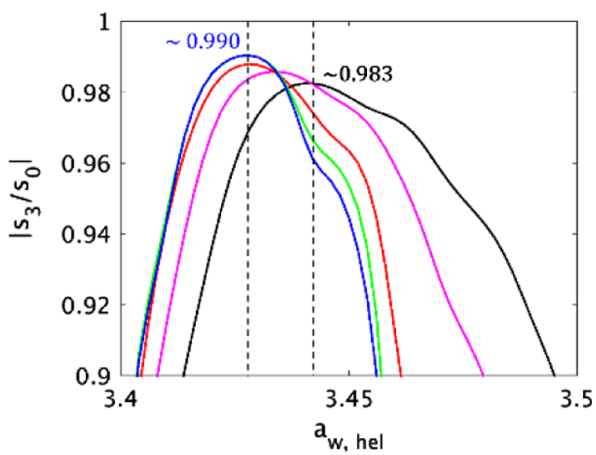

(b)

FIG. 4. Simulation results of the existing method (black lines) and the modified reverse tapering method with 1 (magenta lines), 2 (red lines), 3 (green lines), and 4 (blue lines) untapered planar undulators vs $a_{w, \text { hel }}$. (a) Total radiation power and (b) degree of circular polarization. 


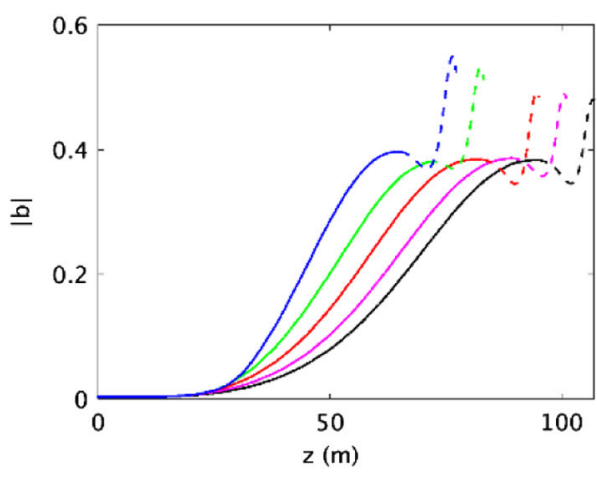

(a)

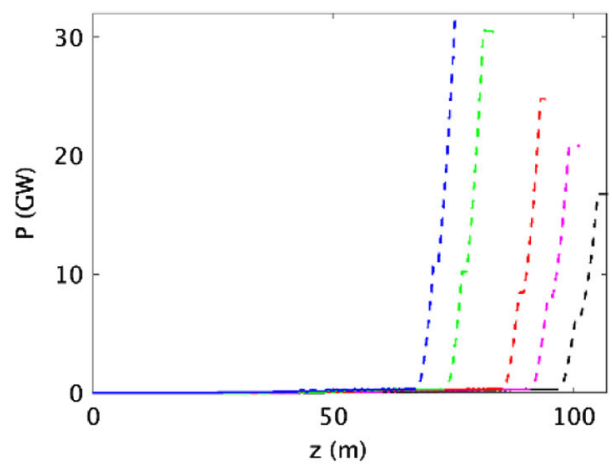

(b)

FIG. 5. Simulation results of the existing method (black lines) and the modified reverse tapering method with 1 (magenta lines), 2 (red lines), 3 (green lines), and 4 (blue lines) untapered planar undulators along the planar undulator line (solid lines) and the helical undulator line (dashed lines). (a) Bunching factor and (b) radiation power.

undulator line. A detailed analysis will be given in the next section.

The bunching factor and the radiation power (Fig. 5) along the whole undulator line were obtained for the five cases with the optimal helical undulator parameters obtained from Fig. 4. As $N_{\mathrm{UT}}$ increased, $L_{\text {sat }}$ in the planar undulator line decreased, and the bunching factor at the end of the helical undulator line increased [Fig. 5(a)]. Because of this higher value of the bunching factor, the total radiation power $P_{\text {tot }}$ in the helical undulator line increased faster, and the final power also increased higher as $N_{\mathrm{UT}}$ increased [Fig. 5(b)].

\section{THE EFFECT OF FREQUENCY SHIFT}

In this section, the effect of the frequency shift in the planar undulator line to the radiation power and the bunching factor will be analyzed by observing the bandwidth and the central frequency of the linearly polarized radiation. The frequency in this section is defined as follows [22]:

$$
E_{\mathrm{ph}}(\mathrm{eV})=\frac{h c}{\lambda} \simeq \frac{1240}{\lambda(\mathrm{nm})},
$$

where $h$ is the Planck constant, $c$ is the speed of light, and $\lambda$ is the wavelength of the radiation. The resonance wavelength of undulator is given by [21]

$$
\lambda_{r}=\frac{\lambda_{u}}{2 \gamma^{2}}\left(1+a_{w}{ }^{2}\right)
$$

where $\lambda_{u}$ is the undulator period, $\gamma$ is the relativistic factor, and $a_{w}$ is the rms undulator parameter.

As the first step for the analysis, the spectra of the radiation for the five cases in Fig. 5 are plotted in Fig. 6. The spectra are gradually widened along the propagation distance $z$. When the existing method $\left(N_{\mathrm{UT}}=0\right)$ is used [Fig. 6(a); black line in Fig. 5], the boundary value of the spectrum at the low energy side (upper part of the graph) followed the resonance frequency of each undulator [white stair shape line, Eq. (4) with $\lambda=\lambda_{r}$ ], and the central frequency also shifted away from the resonance frequency of the first planar undulator. The spectrum was slowly widened as $N_{\text {UT }}$ increased [Figs. 6(b)-6(e)]. For moredetailed analysis, the bandwidth and the central frequency are plotted in Fig. 7.

In all cases, the bandwidth started at $2 \%$, decreased to a minimum, and then increased until the end of the planar undulator line [Fig. 7(a)]. As $N_{\mathrm{UT}}$ increased, the bandwidth decreased faster and then increased slower. The minimum value of the bandwidth is lowered with large $N_{\mathrm{UT}}$. When the existing method [black solid line, Fig. 7(a)] was used, the minimum bandwidth was $0.60 \%$ and increased to $1.25 \%$ at the end of the planar undulator. When the modified reverse tapering method is used, the minimum bandwidth was $0.50 \%$ for $N_{\mathrm{UT}}=1,0.38 \%$ for $N_{\mathrm{UT}}=2$, $0.29 \%$ for $N_{\mathrm{UT}}=3$, and $0.25 \%$ for $N_{\mathrm{UT}}=4$ [Fig. $7(\mathrm{a})$ ]. The bandwidth at the end of the planar undulator line was $1.02 \%$ for $N_{\mathrm{UT}}=1,0.71 \%$ for $N_{\mathrm{UT}}=2,0.41 \%$ for $N_{\mathrm{UT}}=3$, and $0.30 \%$ for $N_{\mathrm{UT}}=4$ [Fig. 7(a)].

The behavior in the growth of the bunching factor and the radiation power in the helical undulator line (dashed lines, Fig. 5) can be explained from the bandwidth change [Fig. 7(a)]. The bandwidth at the entrance of the helical undulator line decreased as $N_{\text {UT }}$ increased [Fig. 7(a)], although $P_{\text {lin }}$ and the bunching factor at the entrance of the helical undulator line for all cases [from (0) to (4) in Fig. 3] are very similar. This means that the spectral density nearby the central frequency increased as $N_{\mathrm{UT}}$ increased. The power of seed radiation will be higher with the large $N_{\text {UT }}$. Therefore, the bunching factor and $P_{\text {tot }}$ in the helical undulator are growing faster than the existing method when the modified reverse tapering method is used.

The central frequency of the spectra [Fig. 7(b)] showed the same trend as the bandwidth. When the existing method was used, the central frequency increased after the sixth undulator 


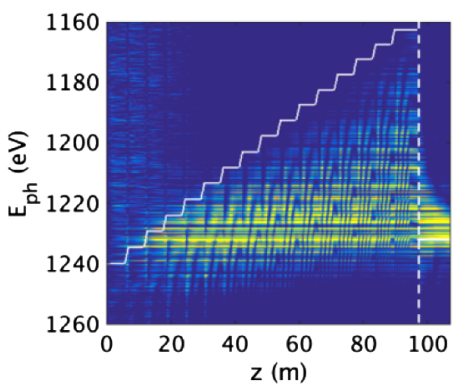

(a)

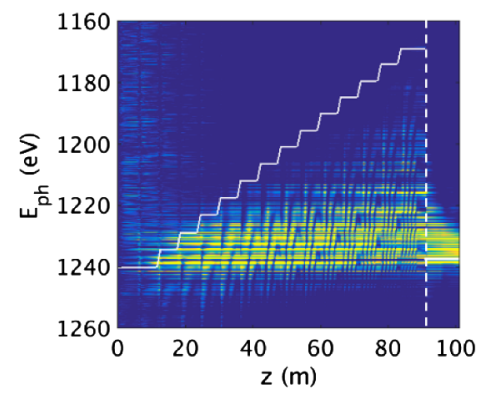

(b)

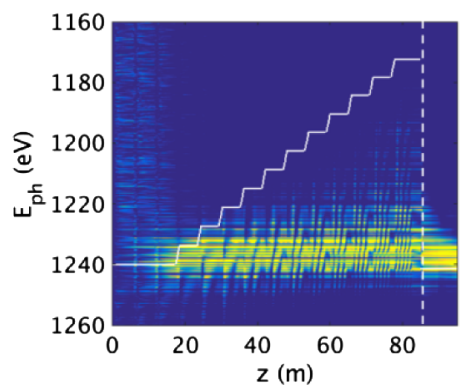

(c)

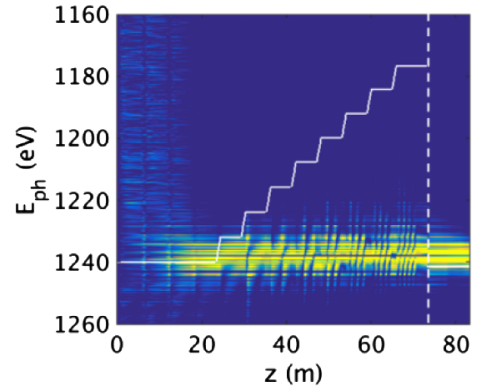

(d)

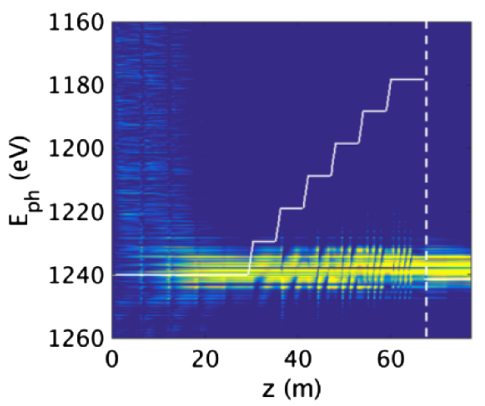

(e)

FIG. 6. Spectrum along the planar undulator line and the helical undulator line in Fig. 5 for (a) the existing method ( $\left.N_{\mathrm{UT}}=0\right)$ and the modified reverse tapering method with (b) $N_{\mathrm{UT}}=1$, (c) $N_{\mathrm{UT}}=2$, (d) $N_{\mathrm{UT}}=3$, and (e) $N_{\mathrm{UT}}=4$. White line (stair shape): resonance frequency of the planar undulator. The white dashed line denotes the end of the planar undulator line. The right side of the white dashed line is the helical undulator line. $z$ denotes the propagation distance of the radiation pulse in the whole undulator line.

( $z=33 \mathrm{~m})$. For the modified reverse tapering method with $N_{\text {UT }} \geq 3$, the central frequency is fixed to $1.237 \mathrm{keV}$, which is almost the same as the resonance frequency of the first planar undulator along the planar undulator line. This is the reason for the phenomena [Fig. 4(a)] in which the helical undulator parameter $a_{w \text {,hel }}$ to get the maximum $P_{\text {tot }}$ moved toward planar undulator parameter $a_{w}$ of the first planar undulator as $N_{\text {UT }}$ increased, and the maximum $P_{\text {tot }}$ is obtained at the same $a_{w, \text { hel }}$ for $N_{\mathrm{UT}} \geq 3$.

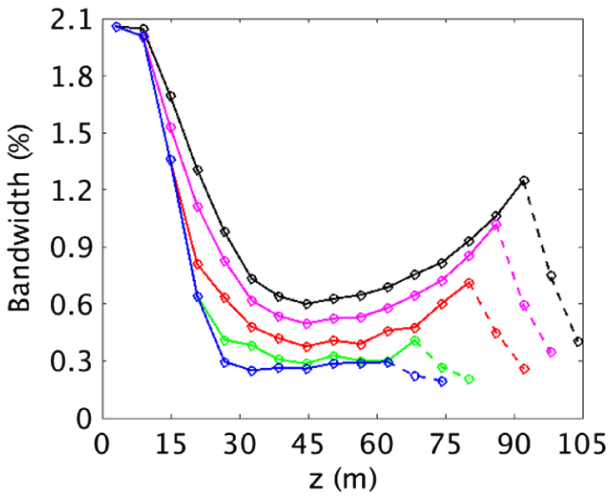

(a)
Theoretically, the central frequency of the radiation was predicted to be fixed at the resonance frequency of the first planar undulator and will not be shifted along the propagation in the planar undulator line when the strong reverse taper strength $(\beta<0$ and $|\beta| \gg 1)$ is applied [12]. However, such a strong reverse taper strength cannot be applied for the existing method, since the bunching factor is too much suppressed [black line, Fig. 3(c)]. On the other hand, in the modified reverse tapering method, a strong

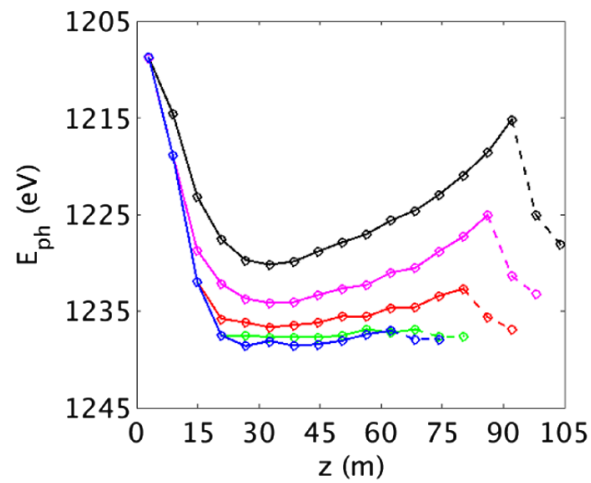

(b)

FIG. 7. (a) Bandwidth and (b) the central frequency of radiation along the planar undulator line (solid lines) and the helical undulator line (dashed lines) in simulation results (Fig. 6) using the existing method (black lines) and the modified reverse tapering method with $N_{\mathrm{UT}}=1$ (magenta lines), $N_{\mathrm{UT}}=2$ (red lines), $N_{\mathrm{UT}}=3$ (green lines), and $N_{\mathrm{UT}}=4$ (blue lines). $z$ is the propagation distance of the electron beam in the undulator line. 


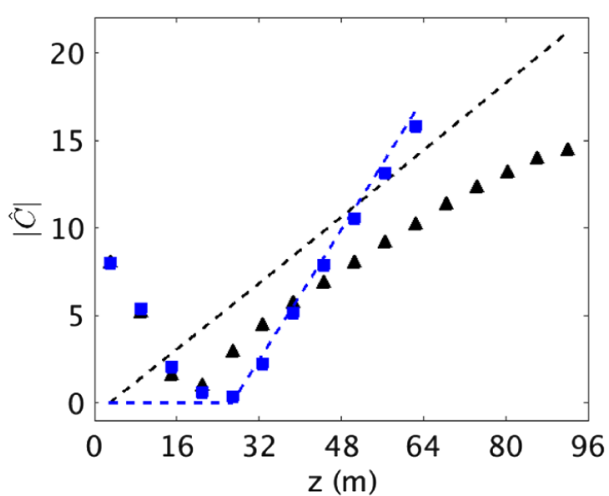

(a)

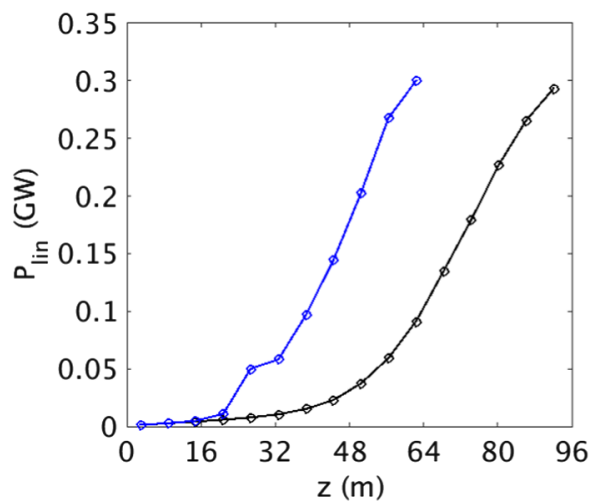

(b)

FIG. 8. (a) The magnitude of the normalized detuning parameter without a frequency shift (dashed lines) and the simulation results with black triangles (existing method) and blue square markers (modified reverse tapering). (b) The linearly polarized radiation power for the existing method (black lines) and the modified reverse tapering method with $N_{\mathrm{UT}}=4$ (blue lines) along the planar undulator line.

reverse taper strength $(\beta<0$ and $|\beta| \gg 1)$ can be applied and has to be applied to suppress the growth of the linearly polarized radiation power [Fig. 3(a)]. The central frequency of the radiation is fixed close to the resonance frequency of the first undulator due to this strong reverse taper strength as shown in Fig. 7(b). As a conclusion, the frequency shift in Refs. [14-16] due to the weak reverse taper strength in the reverse tapered undulator [17] can be prevented by using the modified reverse tapering method with a strong reverse taper strength.

Now we can explain the rapid growth of the bunching factor in the planar undulator line [solid line, Fig. 5(a)] for the modified reverse tapering method by considering the normalized detuning parameter and the linearly polarized radiation power (Fig. 8). The normalized detuning parameter $\hat{C}$ is defined as [21]

$$
\begin{aligned}
\hat{C}(z) & =\left(\frac{1}{\lambda_{u}}-\frac{1}{\lambda(z)} \cdot \frac{\left[1+a_{w}^{2}(z)\right]}{2 \gamma^{2}}\right) \frac{\lambda_{u}}{2 \rho} \\
& =\left(1-\frac{\lambda_{r}(z)}{\lambda(z)}\right) \frac{1}{2 \rho},
\end{aligned}
$$

where $\lambda_{u}$ is the undulator period, $\lambda$ is the wavelength of the electromagnetic wave, $a_{w}$ is the rms undulator parameter, $\gamma$ is the relativistic factor, $\rho$ is a FEL parameter (Pierce parameter), and $\lambda_{r}$ is the resonance wavelength of the undulator as given in Eq. (5).

If we assume that the central frequency of the radiation does not shift, $\lambda$ in Eq. (6) can be considered as a constant value which is the resonance wavelength of the first planar undulator. Then, the amplitudes of $\hat{C}$ under the assumption can be simply plotted by using Eq. (6) for the existing method [black dashed line, Fig. 8(a)] and the modified reverse tapering method with $N_{\mathrm{UT}}=4$ [blue dashed line, Fig. 8(a)].
From the simulation results [Fig. 7(b)], the amplitudes of $\hat{C}$ were calculated for the existing method [black triangles, Fig. 8(a)] and the modified reverse tapering method with $N_{\text {UT }}=4$ [blue squares, Fig. 8(a)]. For the existing method, the normalized detuning parameters from the simulation results [black triangles, Fig. 8(a)] are lower than the prediction [black dashed line, Fig. 8(a)]. In contrast, for the modified reverse tapering method with $N_{\mathrm{UT}}=4$, the normalized detuning parameters from the simulation results [blue squares, Fig. 8(a)] and prediction [blue dashed line, Fig. 8(a)] are matched well, because the central frequency of the radiation in the simulation is fixed close to the resonance frequency of the first undulator [see the blue line after $z=30 \mathrm{~m}$ in Fig. 7(b)]. Note that the detuning parameter of the modified reverse tapering at the end of the planar undulator line is higher than that of the existing method.

The authors in Ref. [12] predicted that the strong reverse taper strength will prevent the radiation power growth. However, for the modified reverse tapering method [blue line, Fig. 8(b)] with $N_{\mathrm{UT}}=4$, the linearly polarized radiation power $\left(P_{\text {lin }}\right)$ grows faster than the existing method [black line, Fig. 8(b)]. The radiation power is still growing in spite of the strong reverse taper strength in the modified reverse tapering method.

When the large negative detuning is applied $(\hat{C}<0$ and $|\hat{C}| \gg 1)$, the bunching factor is connected by the normalized detuning parameter and the linearly polarized radiation power in the high-gain FEL using the one-dimensional, cold beam [12]:

$$
|b|^{2} \simeq|\hat{C}|^{2} \cdot \frac{P_{\text {lin }}}{\rho P_{\text {beam }}} .
$$

where $b$ is the bunching factor, $P_{\text {lin }}$ is the linearly polarized radiation power, $\rho$ is the FEL parameter, and $P_{\text {beam }}$ is the electron beam power. As another conclusion, the bunching 
factor in the planar undulator line is growing faster than the existing method according to Eq. (7), since both the normalized detuning parameter [Fig. 8(a)] and the linearly polarized radiation power [Fig. 8(b)] are growing faster when the modified reverse tapering method is used.

\section{CONCLUSION AND DISCUSSION}

The existing method causes the frequency shift in the radiation, but the modified reverse tapering method does not. The modified reverse tapering method makes the bunching factor to increase quickly in the planar and helical undulator line, so that the total radiation power also increases. The modified reverse tapering method reduces the saturation length also compared to the existing method, even though the linearly polarized radiation power and the bunching factor are similar with the existing method at the end of the planar undulator line.

The modified reverse tapering method consists of the combination of untapered and linearly reverse tapered planar undulators which may be regarded as an approximation of a quadratic tapering profile. However, when reverse tapering has a quadratic profile, $\beta$ is also changed gradually, and the frequency is shifted in an early stage of reverse tapering (simulation results are not shown in this paper). The final radiation power from a quadratic profile is lower than that of the modified reverse taper method.

In the PAL-XFEL case, when $N_{\mathrm{UT}}=4$ was used in the modified reverse tapering method, the length of the planar undulator line was reduced about $30 \mathrm{~m}$ from the required length for the existing method, and the radiation power of the soft $\mathrm{x}$-ray pulse $(1.24 \mathrm{keV})$ reached to $31.2 \mathrm{GW}$ with 0.99 degree of circular polarization.

In this paper, we have considered the soft $\mathrm{x}$ ray only, in which the circular polarized radiation is strongly demanded. In the case of different photon energy such as a hard $\mathrm{x}$ ray or UV, the modified reverse tapering method can also be applied, in principle. However, in the UV region, we need to use short undulators, because the saturation length is rather shorter than the soft or hard x-ray cases. For the hard x ray, there is a very convenient technique to control the polarization developed in the SPring-8 Angstrom Compact FreeElectron Laser [13].

\section{ACKNOWLEDGMENTS}

This research has been supported by the Basic Science Research Program through the National Research Foundation of Korea (NRF-2016R1D1A1B03930349). This research has been supported in part by Global Research Laboratory Program [Grant No. 2009-00439] and by Max Planck POSTECH/KOREA Research Initiative Program [Grant No. 2016K1A4A4A01922028] through the National Research Foundation of Korea (NRF) funded by Ministry of Science, ICT \& Future Planning.
[1] W. Ackermann et al., Operation of a free-electron laser from the extreme ultraviolet to the water window, Nat. Photonics 1, 336 (2007).

[2] P. Emma et al., First lasing and operation of an ångstromwavelength free-electron laser, Nat. Photonics 4, 641 (2010).

[3] T. Ishikawa et al., A compact X-ray free-electron laser emitting in the sub-ångström region, Nat. Photonics 6, 540 (2012).

[4] H. S. Kang, K.-W. Kim, and I. S. Ko, Status of the PALXFEL construction, in Proceedings of the 6th International Particle Accelerator Conference, Richmond, USA, 2015 (JACoW, CERN, Geneva, 2015), p. 2439.

[5] Swiss FEL Conceptual Design Report, edited by R. Ganter, PSI Bericht No. 10-04, 2012.

[6] M. Altarelli et al., Technical Design Report No. DESY 2006-097, 2007.

[7] G. Geloni, V. Kocharyan, and E. Saldin, Report No. DESY10-252, 2010.

[8] G. Geloni, V. Kocharyan, and E. Saldin, Report No. DESY11-083, 2011.

[9] G. Geloni, V. Kocharyan, and E. Saldin, Report No. DESY11-096, 2011.

[10] E. A. Schneidmiller and M. V. Yurkov, An option of frequency doubler at the European XFEL for generation of circularly polarized radiation in the wavelength range 1-2.5 nm, in Proceedings of the 32nd Free Electron Laser Conference, Malmö, Sweden (JACoW, CERN, Geneva, 2010), p. 123.

[11] E. Allaria, Y. Ding, P. J. Emma, Z. Huang, H.-D. Nuhn, M. Rowen, J. Welch, and J. Wu, Studies for polarization control at LCLS, in Proceedings of the 33rd Free Electron Laser Conference, Shanghai, China, 2011 (JACoW, CERN, Geneva, 2011), p. 31.

[12] E. A. Schneidmiller and M. V. Yurkov, Obtaining high degree of circular polarization at X-ray free electron lasers via a reverse undulator taper, Phys. Rev. ST Accel. Beams 16, 110702 (2013).

[13] M. Suzuki, Y. Inubushi, M. Yabashi, and T. Ishikawa, Polarization control of an X-ray free-electron laser with a diamond phase retarder, J. Synchrotron Radiat. 21, 466 (2014).

[14] J. MacArthur, A. Marinelli, A. Lutmann, T. Maxwell, H.-D. Nuhn, D. Ratner, and Z. Huang, Investigation of reverse taper to optimize the degree of polarization for the Delta undulator at the LCLS, in Proceedings of the 36th International Free Electron Laser Conference, Basel, Switzerland, 2014 (JACoW, CERN, Geneva, 2014), p. 465.

[15] E. A. Schneidmiller and M. V. Yurkov, Reverse undulator tapering for polarization control at XFELs, in Proceedings of the 7th International Particle Accelerator Conference, Busan, Korea, 2016 (JACoW, CERN, Geneva, 2016), p. 722 .

[16] J. MacArthur, A. Marinelli, A. Lutmann, H.-D. Nuhn, and Z. Huang, Energy spread constraints on field suppression in a reverse tapered undulator, in Proceedings of the 37th International Free Electron Laser Conference, Daejeon, Korea, 2015 (JACoW, CERN, Geneva, 2015), p. 597.

[17] Z. Huang and G. Stupakov, Free electron lasers with slowly varying beam and undulator parameters, Phys. Rev. ST Accel. Beams 8, 040702 (2005). 
[18] C. H. Shim, I. S. Ko, and Y.W. Parc, New soft X-ray undulator line using $10 \mathrm{GeV}$ electron beam in PAL-XFEL, in Proceedings of the 37th International Free Electron Laser Conference, Daejeon, Korea, 2015 (JACoW, CERN, Geneva, 2015), p. 237.

[19] C. H. Shim, I. S. Ko, Y. W. Parc, and J.-H. Han, Soft X-ray FEL simulation in PAL-XFEL, J. Korean Phys. Soc. 67, 1301 (2015).

[20] S. Reiche, GENESIS 1.3: a fully 3D time-dependent FEL simulation code, Nucl. Instrum. Methods Phys. Res., Sect. A 429, 243 (1999).
[21] E. L. Saldin, E. A. Schneidmiller, and M. V. Yurkov, The Physics of Free Electron Lasers (Springer, Berlin, 1999).

[22] E. Hecht, Optics (Addison-Wesley, New York, MA, 2002), 4th ed.

[23] C. H. Shim, I. S. Ko, J.-H. Han, and Y. W. Parc, A research on the reverse tapering method to gain high power polarized photon beam with fixed wavelength, in Proceedings of the 6th International Particle Accelerator Conference, Richmond, USA, 2015 (JACoW, CERN, Geneva, 2015), p. 1678. 